\title{
Correcting the ADCS Jitter Induced Blurring in Small Satellite Imagery
}

\author{
D. Llaveria, A. Camps, Fellow, IEEE, H. Park, Senior Member, IEEE
}

Earth Observation missions have increasingly demanding requirements, i.e. higher spatial resolutions and SNR, and lower revisit times, etc. High resolution images have to be captured with precise pointing accuracy which is achieved by the satellite's Attitude Determination and Control System (ADCS). This subsystem embeds multiple sensors and actuators capable of changing the satellite attitude by changing the torque applied to the platform. In small satellites, magnetorquers, and more recently reaction wheels as well, are the most frequently used actuators. These components fulfill their main purpose, but exhibit some drawbacks, notably a non-constant jitter that provokes non-desired oscillations of the spacecraft, which may result in blurred images. This work presents a deblurring methodology that uses data coming from the ADCS sensors to infer a spatially variant point-spread function. Moreover, the impact of having a lower temporal resolution than the ideal on the sensors is analyzed and a method is presented to improve it.

Keywords - Small Satellites, Imaging Deblurring, Earth Observation, Optical sensors.

\section{INTRODUCTION}

$\mathrm{E}$ ARTH Observation (EO) missions have increasingly demanding requirements. High resolution imagers are nowadays being embarked on small satellites to fulfill these specifications. Thanks to the miniaturization of these sensors, they could be embarked in satellites as small as $3 \mathrm{U}$ Cubesats while achieving 3-meter resolution images [1]. These high-resolution imagers demand a precise pointing accuracy. Satellites' attitude is controlled by the Attitude Determination and Control System (ADCS). ADCS gathers data from its sensors (e.g. gyroscopes, magnetometers, Sun sensors, star trackers, etc.), that data is used to determine the satellite attitude. Then the subsystem uses its actuators (e.g. reaction wheels, magnetorquers, etc.) to change it [2], [3].

As in large satellites, reaction wheels and magnetorquers are becoming common in CubeSats. The controller of the reaction wheels induces small fluctuations on the angular velocity around the nominal value, and magnetorquers' current also fluctuates around the nominal value which is controlled by the PWM (Pulse Width Modulation) controller. Due to the finite number of levels of the controllers and discretization errors, some fluctuations are induced on the torque generated. Having a fluctuating torque on the actuators induces a jitter on the satellite's attitude. Furthermore, if this jitter is induced when the optical sensor is capturing an image, it generates a movement of the Ground Field of View (Instantaneous GFOV), which translates into blurred images.

This document has been submitted on March 31, 2020. This work has been supported by project "Sensing with pioneering opportunistic techniques-SPOT," Spanish Agencia Estatal de Investigación Grant RTI2018-099008-B-C21 and EU ERDF funds, and UPC-CommSensLab María de Maeztu Unit Grant MDM-2016-0600.

D.L. Author is with the Universitat Politècnica de Catalunya (UPC), Barcelona, 08034, Spain (e-mail: david.1laveria@upc.edu).
Figure 1 shows a sample magnetic field reading of one of the magnetometers onboard the ${ }^{3} \mathrm{Cat}-4$ ADCS [4] board when the magnetorquers are working at a constant value. As it can be seen, the magnitude of the magnetic field has some periodic oscillations. In this example, the largest fluctuations occur approximately every $50 \mathrm{~s}$. and last about $9 \mathrm{~s}$. where the oscillations are present. During this period, a typical LEO satellite ground-track can move over $58 \mathrm{~km}$, and therefore, the area affected by this non-ideal behavior of the actuator is large enough to lose several images.

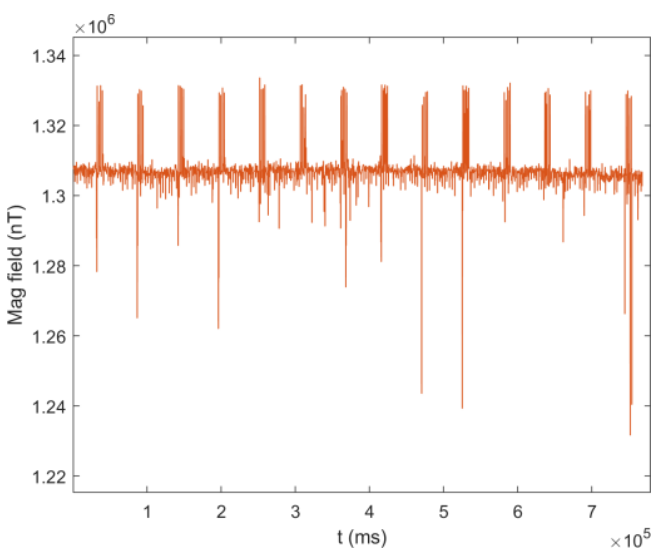

Figure 1. Sample measured magnetic field onboard ${ }^{3}$ Cat -4 ADCS board.

There are methodologies to compensate for the jitter induced by the ADCS actuators. Reaction wheels' non-ideal behavior can be compensated using other actuators such as

A.C. Author is with the Universitat Politècnica de Catalunya (UPC), Barcelona, 08034, Spain (e-mail: camps@tsc.upc.edu).

H.P. Author is with the Universitat Politècnica de Catalunya (UPC), Barcelona, 08034, Spain (e-mail: park.hyuk@ tsc.upc.edu). 
the magnetorquers, as seen in [5]. Although this can be a valid solution for some satellites, it is not feasible for micro and nano satellites. Furthermore, these solutions do not completely solve the problem. Even if an isolation technique is used to reduce the jitter created by the actuators such as reaction wheels, there is a residual satellite attitude jitter [6]. The magnitude of the jitter depends on the actuator itself, and the desired angular speed. The larger the speed, the faster the jitter.

This study presents the effects of simulated displacements during the integration time of the camera, and a method to restore the blurred images using the data coming from the ADCS sensors.

\section{IMAGE BLURRING}

A blurred image $(B)$ is the result of a movement during the integration time of the camera. This image will present smoother edges than the original image $(I)$. Therefore, image blurring can be modelled as a low-pass filter of the image. Therefore, it can be written as the convolution of the original noisy image and a low-pass filter with some Gaussian noise $\left(N \sim N\left(0, \sigma^{2}\right)\right)$ added. Photodetectors' noise is not Gaussian, but for high SNR it can be approximated as an additive Gaussian Noise. The filter is called the point spread function $(P S F)$ or blur kernel.

$$
B=I \otimes P S F+N,
$$

\section{A. Earth Observation blurred images}

Small satellites do not usually have the capability to change its orbital position, but they can change their attitude. Therefore, attitude jitter induces a non-desired rotational movement on the platform that creates a blurred image. Furthermore, satellites present an almost constant linear velocity on its subsatellite point. So, blurring of the EO images is originated by the combination of these two movements. The linear one induces a small blur effect even with high resolution cameras, e.g. with a 3-meter resolution camera, a typical subsatellite point speed of $6.5 \mathrm{~km} / \mathrm{s}$ suffers a displacement of $6.5 \mathrm{~m}$ during an integration time of $1 \mathrm{~ms}$. Therefore, the blurring effect is about two pixels.

However, due to the rotational movement, since satellite's camera is far away from its target, very small rotations result in highly blurred images. The three different rotations, roll, pitch and yaw provoke different blurring effects on the image. With a nadir-looking camera, for short integration times, roll and pitch induce an almost linear blur, but yaw induces a rotational one. As we are talking of low angular velocities, yaw produces a very small blur on the resulting images, but in the case of roll and pitch, the blur can be really noticeable. Figure 2 shows the effect of these three rotations on a 3-meter resolution image of the city of Barcelona [7]. Section III presents a summary of how these blurred images are generated. The plots on the images shows the angular velocity suffered by the platform during the integration time of the camera. As it can be seen in the figure, yaw has a very low impact on the blur. To make it more visible, the yaw blur has been generated with an angular velocity ten times larger than the other two, and the effect is still almost negligible. That is because the yaw rotation induces a rotation on the image and, as they are very low rotation velocities, the impact is very low. On the other hand, for roll and pitch, even a very small rotation provokes large displacements, as it can be seen in Figure 2.

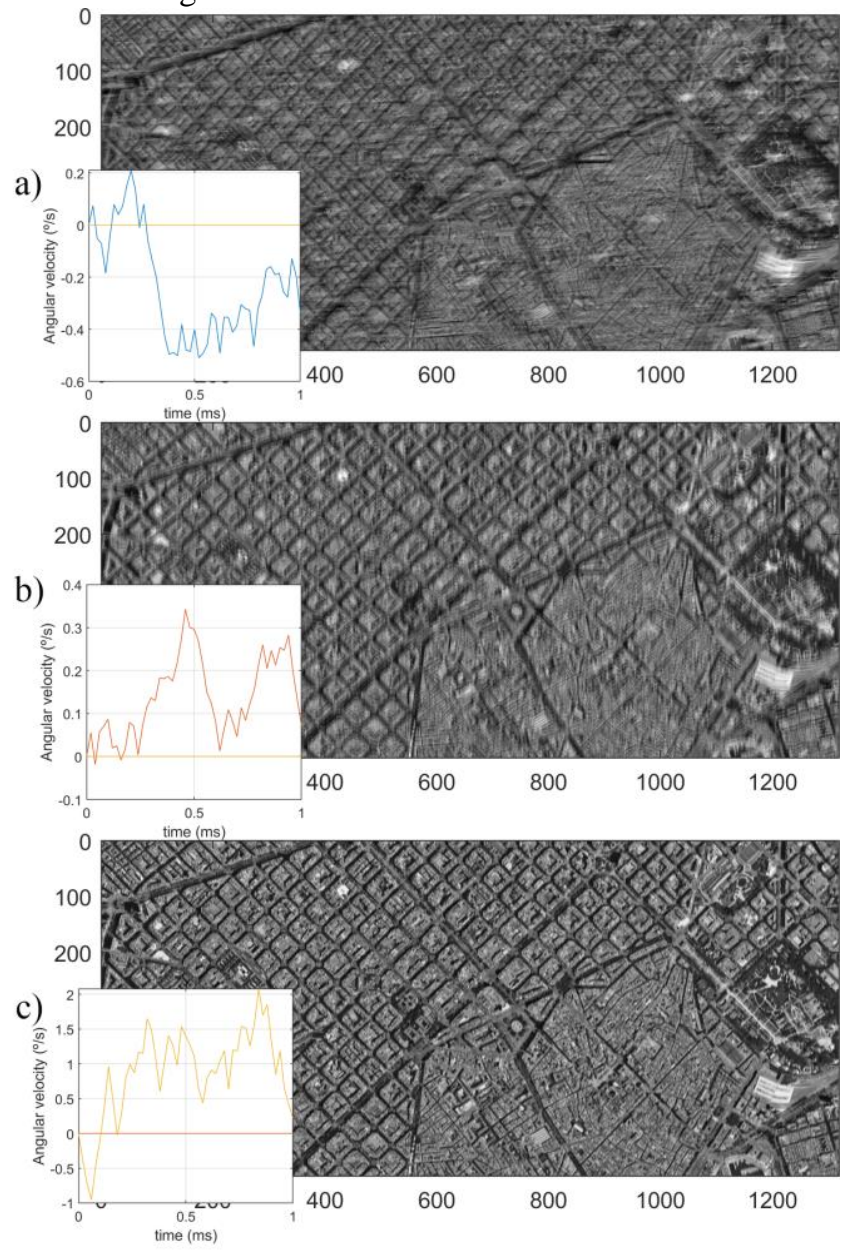

Figure 2. Simulated images blurred by rotation of a) Roll, b) Pitch, c) Yaw.

\section{Deblurring Methodology}

A blurred image can be ideally recovered by a deconvolution. This can be done if the PSF is known and it is constant across the image. However, in a real case these premises do not hold. Due to the camera optics and the different distances for every pixel on the image, the PSF is spatially variant. Moreover, the PSF is unknown.

As mentioned before, the ADCS is equipped with different sensors that gather different data. Gyroscopes measure the angular velocity of the satellite. These data can be used to infer the rotational movement suffered by the camera during the integration time. In [8], a methodology to compute the space variant PSF using the data coming from inertial sensors is presented. This methodology divides the integration times in smaller intervals and compute one PSF 
for each frame. Therefore, a planar homography that relates the pixel coordinates of the projection points at the initial time $\left(u_{0}, v_{0}\right)$ to any other time frame $\left(u_{t}, v_{t}\right)$ is defined:

$$
\begin{aligned}
& H_{t}(d)=K\left(R_{t}+\frac{1}{d} T_{t} \vec{N}^{T}\right) K^{-1}, \\
& \left(u_{t}, v_{t}, 1\right)^{T}=H_{t}(d)\left(u_{0}, v_{0}, 1\right)^{T},
\end{aligned}
$$

being $K$ the intrinsic matrix of the camera, $R_{t}$ the rotation matrix that relates the frame in $t$ to the frame in $t_{0}, T_{t}$ the translation of the camera, $d$ the scene depth, $\vec{N}$ the unit vector orthogonal to the image plane, $u_{t}$ and $v_{t}$ the pixel coordinates in time $t$ and $u_{0}$ and $v_{0}$ the pixel coordinates in the initial time $t_{0}$.

Therefore, the image $(I)$ at the initial time can be transformed to the image $\left(I_{t}\right)$ at time $t$. In matrix form, where images are in vector form, it can be written as follows:

$$
\overrightarrow{I_{t}}=A_{t}(d) \vec{I} \text {. }
$$

Each row of matrix $A_{t}(d)$ contains the weights of the different pixels from $I$ to compute the new image. Therefore, it is a very large sparse matrix. With the images of the different timeframes, the final matrix, $A(d)$, can be computed as the average of them.

$$
A(d)=\frac{1}{M} \sum_{i=0}^{M} A_{i}(d),
$$

where, $M$ is the number of temporal steps to form the image, i.e. $\Delta \mathrm{t}=\tau / M$, and $\tau$ the total exposure time. Each row of $A(d)$ can be seen as a vectorized form of the PSF of each pixel, centered on that pixel.

As seen in (2), two matrices are needed to define the planar homography that leads to the final $A(d)$ matrix. These matrices are the rotation matrix, $R_{t}$, and the translation, $T_{t}$, one. As mentioned at the beginning of this section, the data gathered by the gyroscopes embarked on the ADCS can be useful for deblurring purposes. Following the steps in [9], this data is used to compute both matrices, knowing that the acceleration can be taken as null to compute the translation matrix in the satellite case, and that velocity is constant and it only depends on the orbit altitude, so it is previously known.

Finally, with the spatially variant PSF computed, the blur equation can be solved:

$$
\vec{B}=A(d) \vec{I}+N
$$

by minimizing the following expression:

$$
\min \left(\|\vec{B}-A(d) \vec{I}\|^{2}\right) .
$$

The methodology chosen to solve this minimization problem is the LSQR [8] algorithm, a Least Square solving methodology that uses QR decomposition. This is a well optimized methodology for large sparse matrices, as is the case of $A(d)$ matrix. It has a good computational performance, but their values are not constrained. Therefore, the results could have values outside the range used in this work, 8-bit. This will result in the loss of some pixels. For this work, the pixels with values larger than 255 , are set as the maxim value. On the other hand, negative pixels are set as 0 .

\section{Simulation Methodology}

To assess the performance of this methodology on synthetic Earth Observation images a procedure has been developed and tested. First, the direct problem is simulated due to the lack of real data. To generate the blurred images, the same methodology to compute the blur kernel shown in Section III is used. First, a random walk angular velocity is generated for the three axes (i.e. roll, pitch and yaw) for an integration time of $1 \mathrm{~ms}$ with a timestep of $0.02 \mathrm{~ms}$. Due to the lack of real data, the angular speed is generated by using random steps with a maximum value of $\pm 0.05 \%$, being 0 $\%$ the initial velocity. In order to test the techniques with larger movements than the real ones and to see the effect of non-ideal behaviors, the value has been chosen larger than the typical jitter magnitude. As it can be seen in section V, for lower velocities, the deblurring technique has better results. The initial linear velocity is set to $6.3 \mathrm{~km} / \mathrm{s}$, a typical subsatellite point velocity for LEO satellites. Once both velocities are generated, the $A(d)$ matrix is computed, and the blurred image is simulated using (6).

On the other hand, for the inverse problem, different cases have been tested. The $A(d)$ matrix to perform the deblurring has been computed using a larger time step, the angular velocity used for the direct problem is decimated to fit to this time step before using it. Additionally, in some cases, some noise is added to the angular velocity, to simulate the effect of the noise on the readings.

Figure 3 shows an example of the angular velocity simulated to generate the direct problem (left) and the one used for the inverse problem (right). In this example, to generate the blur kernel to restore the image, the temporal resolution used is $0.04 \mathrm{~ms}$. In this case, it can be seen that the readings for the inverse problems are not exactly the same than the ones used for the direct problem, this is due to the noise added to the angular velocity readings.
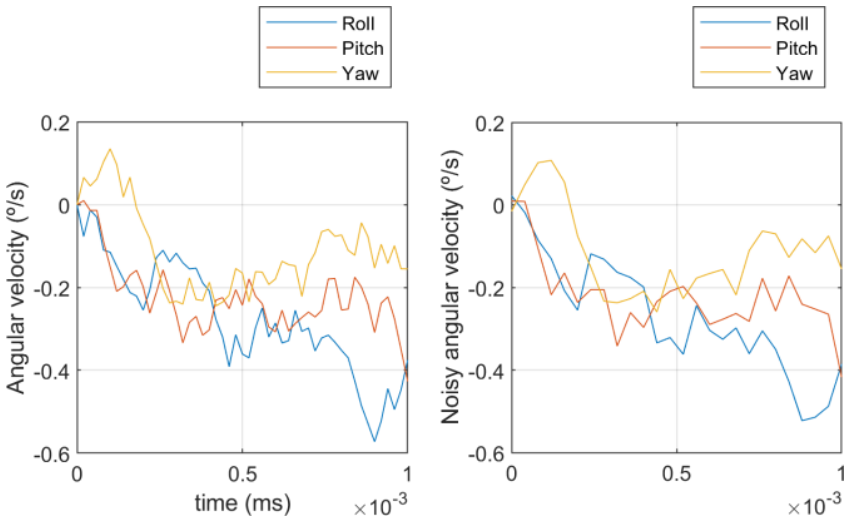

Figure 3. Example of the simulated angular velocity for the direct and the inverse problem.

To measure the quality of the restoration itself, different 
metrics are used, cross-correlation, Mean Squared Error (MSE), Peak Signal-to-Noise Ratio (PSNR), structural similarity index measure (SSIM) and the sharpness degree. The cross-correlation, the, MSE, the PSNR and the SSIM values are computed between the original image, the one used to compute the blurred one, and the final reconstructed image. The sharpness degree is computed for both, the blurred and the reconstructed, images and then, its values are compared to see the increase of sharpness. Furthermore, as it will be seen in the next section, some artifacts appear on the margins of the image, therefore the metrics to assess the quality of the restoration are computed eliminating some pixels of all the edges of the images.
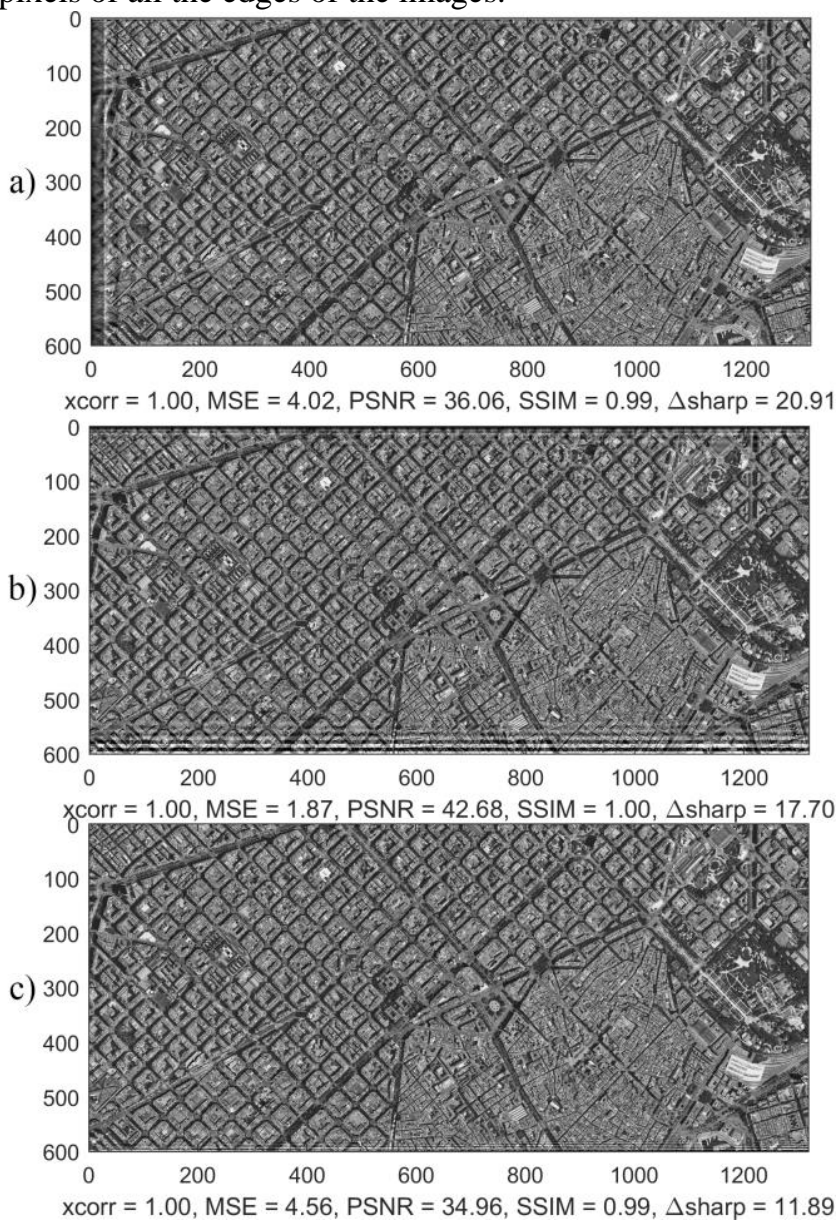

Figure 4. Deblurred images for the a) Roll, b) Pitch and c) Yaw cases in Figure 2

\section{RESULTS}

\section{A. Roll, pitch and yaw cases}

Using the steps described in Sections II and III, the methodology has been tested on different cases. First, the images from Figure 2 have been restored using the same time step $(0.02 \mathrm{~ms})$ than the one used on the direct problem, i.e. no data is lost, and with zero noise. Figure 4 shows the restoration results, and the artifacts commented in the previous section. The straight white lines appear due to the loss of information, this effect is called ringing effect. The larger the displacement the wider the white lines. However, looking at the center part of the images the reconstruction looks quite good, the cross-correlation and the SSIM are close to 1 . The three cases show an improvement of the sharpness degree. Table 1 shows the sharpness degree of the original, the blurred and the restored images. The sharpness degree is fully recovered. Although, the yaw case presents some new artifacts that make the image sharper than the original, they have a low impact on the result.

TABLE I

SHARPNESS DEGREE CASE A

\begin{tabular}{lrcc}
\hline \hline & Original & Blurred & Restored \\
\hline Roll & 29.67 & 9.03 & 29.67 \\
Pitch & 29.67 & 11.96 & 29.67 \\
Yaw & 29.67 & 18.17 & 30.07 \\
\hline \hline
\end{tabular}

a)
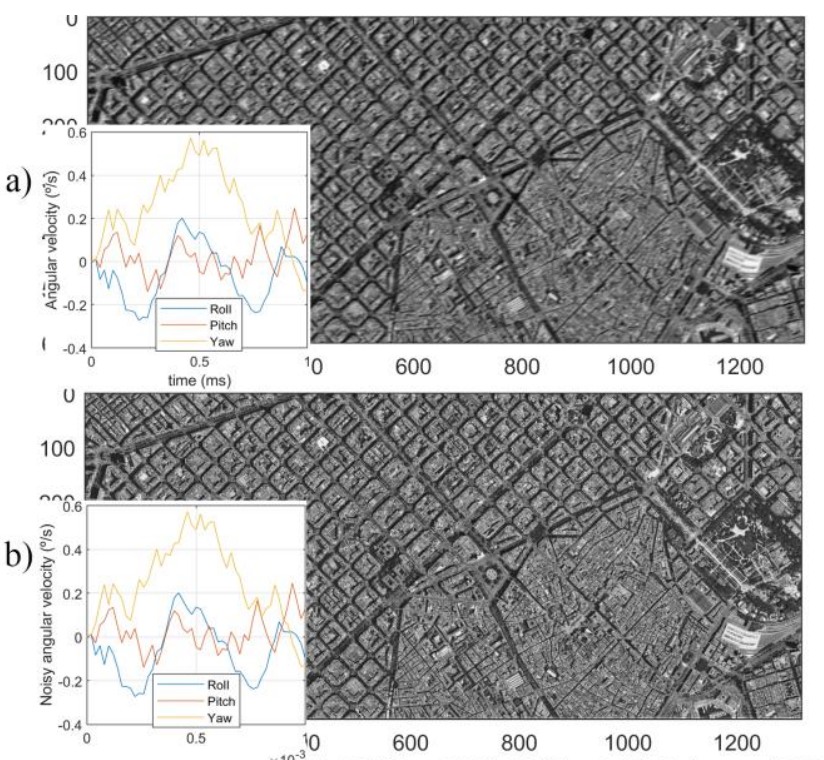

$\mathrm{xcorr}=0.99, \mathrm{MSE}=5.97, \mathrm{PSNR}=32.61, \mathrm{SSIM}=0.98, \Delta$ sharp $=17.69$

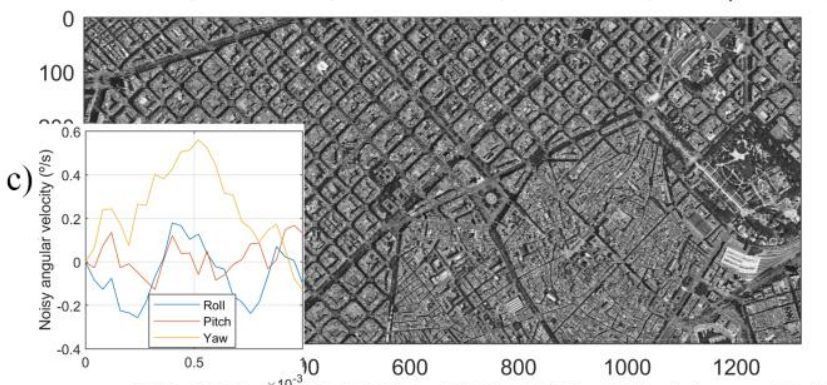

xcorr $=0.95$, MSE $=15.53$, PSNR $=24.31, \operatorname{SSIM}=0.87, \Delta$ sharp $=10.49$

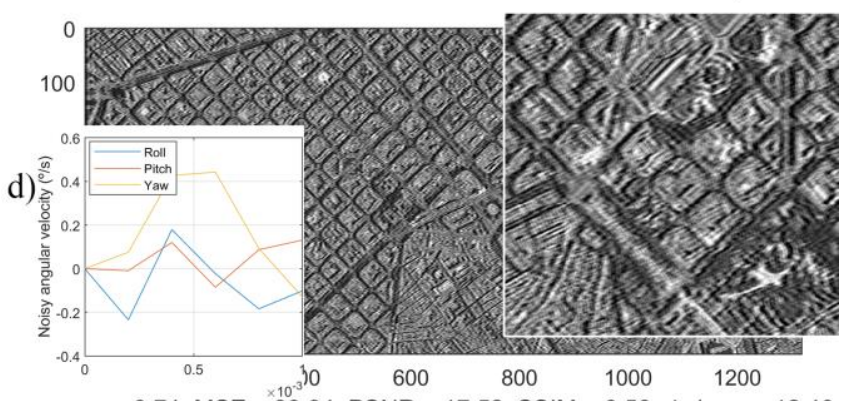

xcorr $=0.74, \mathrm{MSE}=33.94, \mathrm{PSNR}=17.52, \mathrm{SSIM}=0.50, \Delta$ sharp $=12.40$

Figure 5. Three axis rotation and temporal decimation; (a) blurred image, (b) restored image using all the temporal 
samples, (c) restored image using half of the temporal samples, and (d) using a tenth of the temporal samples.

\section{B. Impact of losing temporal samples}

After that, one case using the three movements at once has been simulated and restored using the same time step, it can be seen in Figure 5. Moreover, this case has been used to see the effect of worsening the temporal resolution of the sensors (under-sampling). Figure 5 shows the blurred image (a) and the angular velocity used to generate it, the reconstruction using all the temporal samples (b) with a cross correlation and a SSIM close to 1, and an improvement of the sharpness degree, in that case, larger than the original one, it can be seen in Table II. The reconstruction using only half of the temporal samples (c), the angular velocity used is shown in the plot on the image. In this case the cross-correlation has decreased down to 0.95 and the SSIM to 0.87 . The sharpness degree is also improved, but less than before, now the blur has not been removed completely due to the loss of data. Finally, the reconstruction using only one tenth of the samples (d), the angular velocity plot clearly shows the loss of data for the reconstruction. The result visually seems good, but if we take a closer look, the ringing effect can be seen on all the sharp edges of the image not only on the margins. Now, the cross-correlation is reduced to 0.74 and the SSIM to 0.5. Looking at the sharpness degree, the blur is not fully removed, and although now it is larger than in (c), the reconstructed image is worse. That is because the ringing effect adds more edges than before.

TABLE II

ShARPNESS DEGREE CASE B

\begin{tabular}{lrcc}
\hline \hline Temporal samples & Original & Blurred & Restored \\
\hline Full & 29.67 & 12.42 & 30.10 \\
Half & 29.67 & 12.42 & 22.90 \\
One tenth & 29.67 & 12.42 & 24.82 \\
\hline \hline
\end{tabular}

\section{Impact of sensors' noise}

The impact of the non-ideal readings coming from the gyroscopes is studied. Some noise level has been added to the angular velocity before the restoration. Figure 6 shows the impact of a noise with a standard deviation equal to the $10 \%$ of the maximum velocity measured. An unnoticeable granular effect appears on the restored image (c), worsening the results. The larger the noise level, the more visible the granular effect, and the worse the restoration is. Moreover, the impact of losing temporal samples is higher than before (d), in that case halving the temporal samples makes the artifacts more visible. The blur effect is not fully removed, and the granular effect is more visible along with the ringing effect. Regarding the sharpness degree, Table III, is similar to the previous case, noise has a low impact on this metric.

TABLE III

SHARPNESS DEGREE CASE C

\begin{tabular}{lrcc}
\hline \hline Temporal samples & Original & Blurred & Restored \\
\hline Full & 29.67 & 8.22 & 23.50 \\
Half & 29.67 & 8.22 & 24.39 \\
One tenth & 29.67 & 8.22 & 24.78 \\
\hline \hline
\end{tabular}
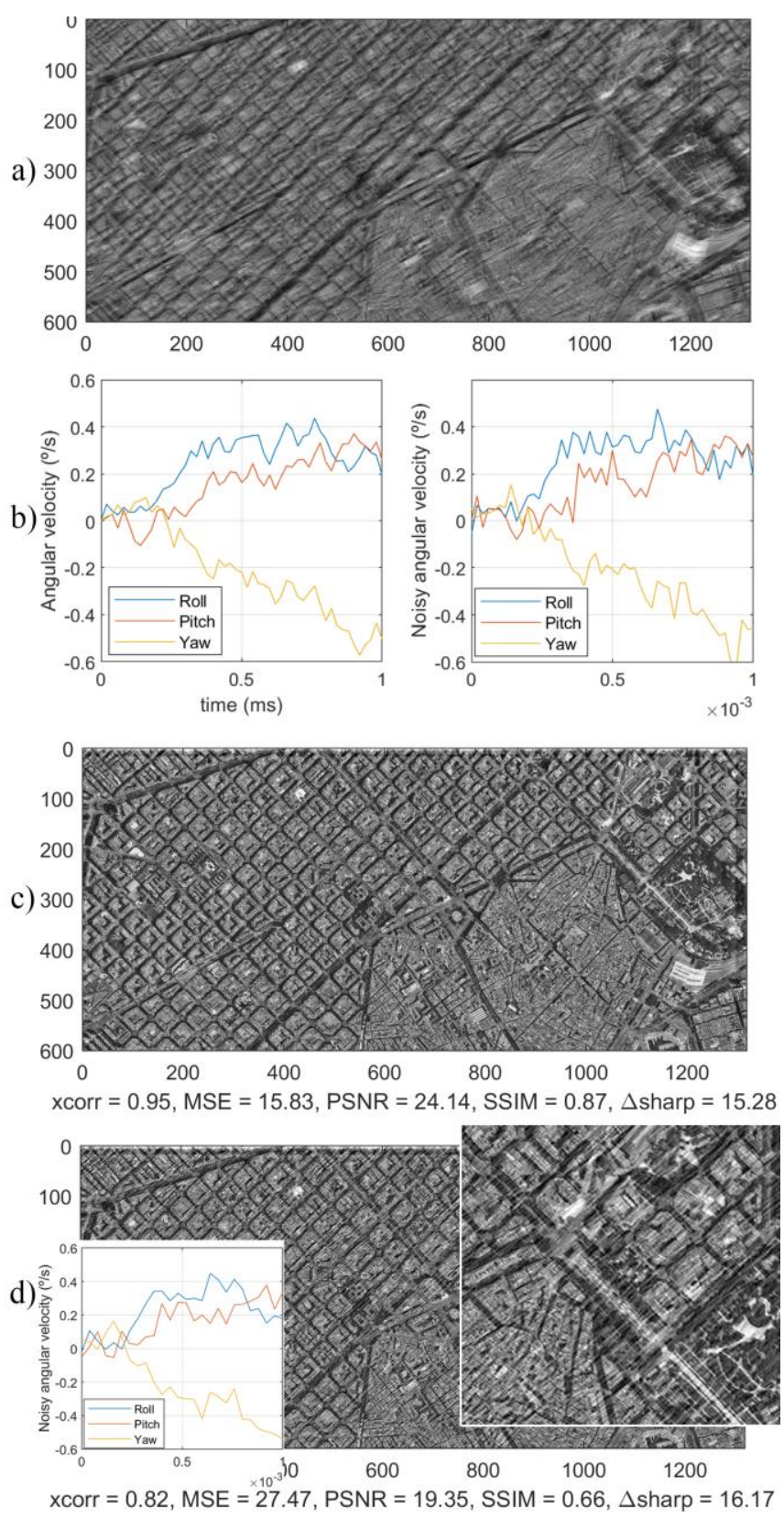

Figure 6. Effect of sensors' noise; (a) blurred image, (b) angular speeds with noise-free(left) and noise (right), (c) restored image using all the temporal samples and (d) restored image using half of the temporal samples.

\section{Impact of higher velocities}

Finally, a high velocity case has been studied. This case velocity is twice the one used in case B. As it can be seen in Figure 7, the restoration is successfully performed achieving a cross-correlation of 0.96 and a SSIM of 0.93 , a bit lower than the case B, but almost perfect, the sharpness degree is a bit lower than the one in the original image, some remaining blur is present on the final image. Furthermore, looking at the margins, more artifacts appear on the resulting image (b), here it can be seen that new pixels have appeared in the bottom of the image, but with not enough information to 
restore them completely. That is because, with higher velocities, more information is lost due to the blurring effect and it cannot be fully restored. Moreover, the effects of losing temporal samples is worst in that case. The restoration using half of the samples (c) has a cross-correlation of 0.93 and a SSIM of 0.71, down from case B. And looking at the restoration using one tenth of the samples (d), it has been completely lost, with a cross correlation and a SSIM close to 0 , there is not enough information to perform the restoration. Furthermore, the sharpness degree, Table IV, for this case is even worse than the blurred image.

a)

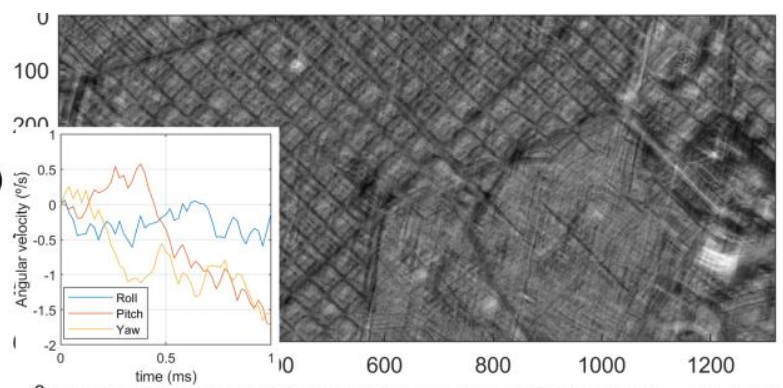

b)

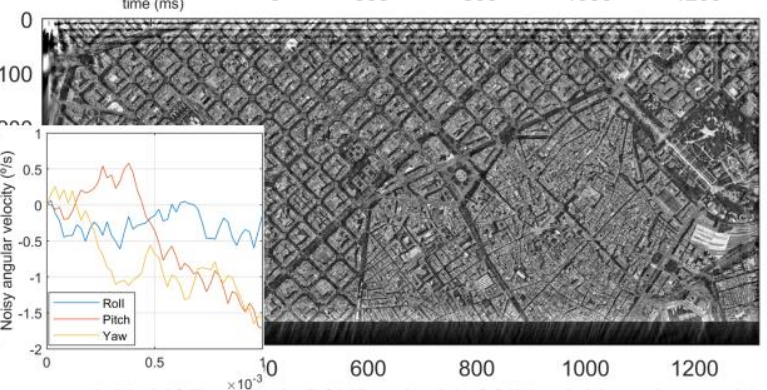

xcorr $=0.96$, MSE $=13.79$, PSNR $=25.34, \operatorname{SSIM}=0.93, \Delta$ sharp $=21.50$
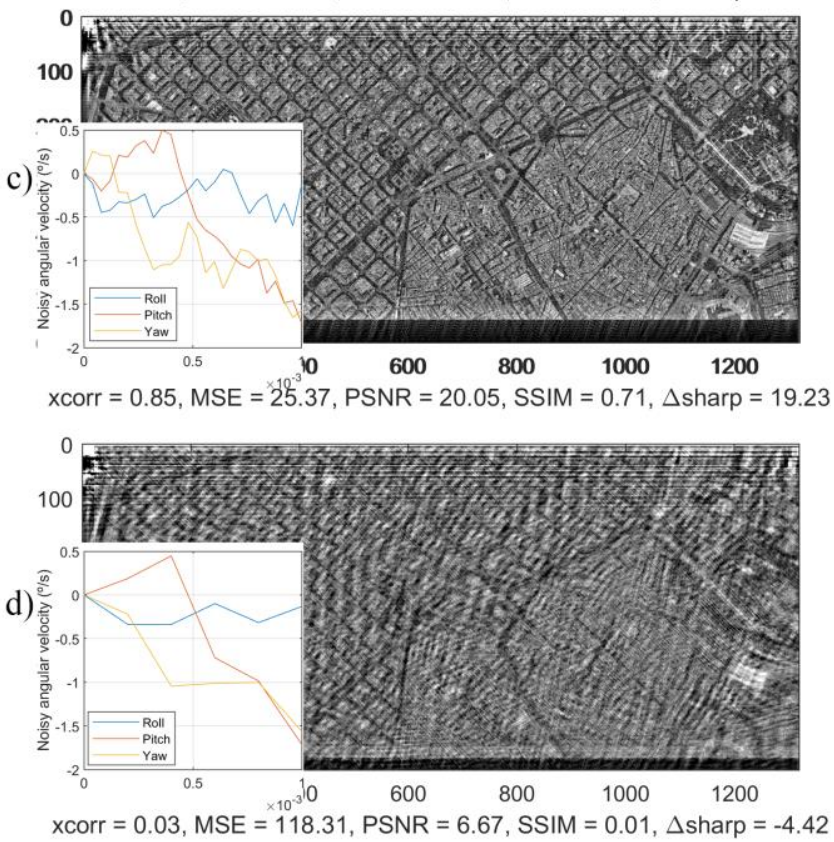

TABLE IV

SHARPNESS DEGREE CASE D

\begin{tabular}{lrcc}
\hline \hline Temporal samples & Original & Blurred & Restored \\
\hline Full & 29.67 & 5.18 & 26.69 \\
Half & 29.67 & 5.18 & 24.41 \\
One tenth & 29.67 & 5.18 & 0.76 \\
\hline \hline
\end{tabular}

\section{IMPROVING THE TEMPORAL INFORMATION}

As seen in section $\mathrm{V}$, the higher the angular velocity the more critical the temporal resolution of the sensors is. In the different angular velocity plots of previous sections, it can be seen that the angular velocity of the platform does not drastically change in each time step. Therefore, the data coming from the gyroscopes can be interpolated to improve the temporal resolution. Although the interpolated values will not be the real ones, they will be closer to the real velocity than the ones used without interpolation.

The next cases studied show the improvement of the reconstruction using linear interpolation for the cases where the data have been previously decimated. Figure 8 shows the results for case $\mathrm{B}$, but with the data improved using linear interpolation. For the image using half of the samples on Figure 5 (c), interpolate the data to have the same step than in the direct problem do not improve the restoration, the metrics are quite similar than before as shown in Figure 8 (a).

On the other hand, for the image on Figure 5 (d), where only one tenth of the temporal samples were used, the interpolation to have the $0.02 \mathrm{~ms}$ timestep results in a significant improvement of the restoration, shown in Figure 8 (b). Now, the cross correlation goes from 0.74 to 0.9 and the SSIM from 0.50 to 0.80 .

Figure 9 shows the same study but for the images of case $\mathrm{D}$, the one with a higher velocity. The improvement for the image in Figure 7 (c), the one where only half of the samples were used, is more significant. In Figure 9 (a) can be seen that the cross correlation goes from 0.85 to 0.95 and the SSIM from 0.71 to 0.82 . But looking the results after interpolating the worst case shown in Figure 7 (d), it can be observed in Figure 9 (b) that the restoration is not perfect, a lot of artifacts have appeared across the image. However, the image that has previously been lost, now is partially recovered. The cross correlation has improved from 0.03 to 0.63 and the SSIM from 0.01 to 0.43 . Moreover, the sharpness degree is almost fully recovered, from 0.76 to 25.65 .

The plots in Figure 8 (c) and Figure 9 (c) show the SSIM for both cases, the decimated and the interpolated as a function of the time step (c).
Figure 7. High velocity case; (a) blurred image, (b) restored image using all the temporal samples, (c) restored image using half the temporal samples, and (d) using a tenth of the temporal samples. 

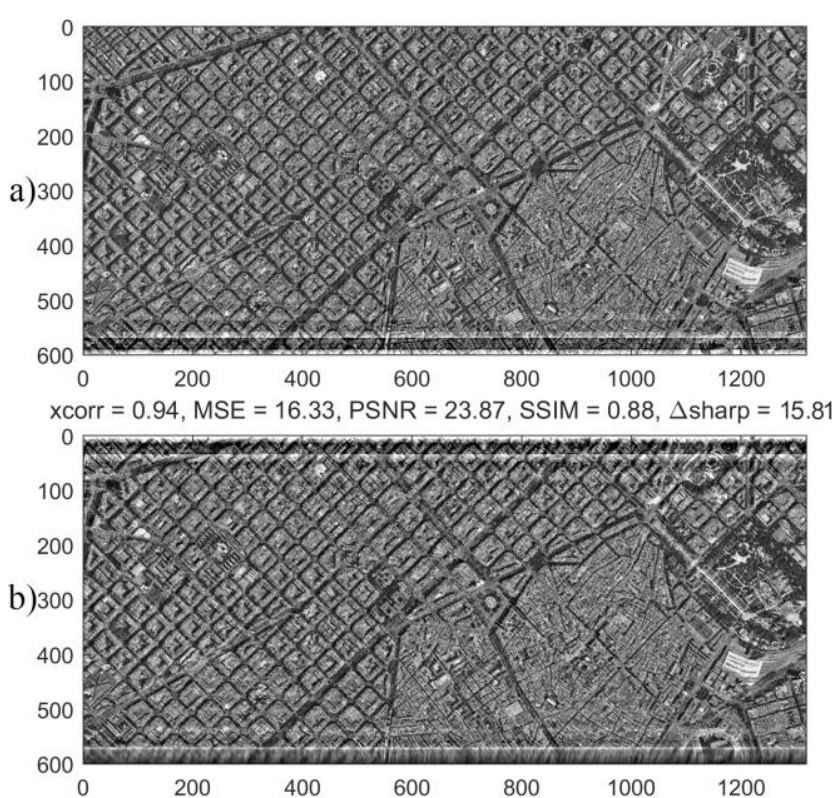

xcorr $=0.90, \mathrm{MSE}=21.63, \mathrm{PSNR}=21.43, \mathrm{SSIM}=0.80, \Delta$ sharp $=13.88$

c)

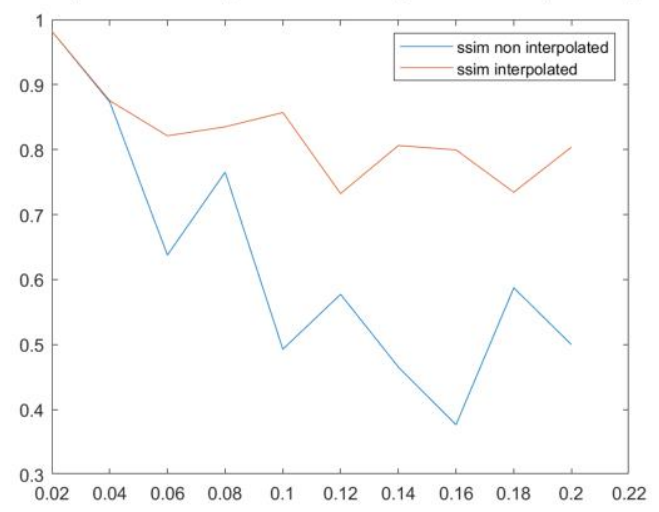

Figure 8. Temporal interpolation case B; (a) Restored image using interpolation from half temporal samples, (b) Restored image using interpolation from on tenth of the temporal samples, and (c) original cross-correlation and interpolated cross-correlation.

Using similar angular velocities as in case B, thirty different simulations have been performed. Each simulation has been done for ten different time steps with and without interpolation. To assess the robustness of the interpolation technique the different metrics for both, interpolated and non-interpolated, cases have been compared and averaged.

Figure 10 shows two of the metrics studied, crosscorrelation (a) and SSIM (b). In each plot can be seen the values of the metrics if no improvement methodology is applied and the values of the metrics when the interpolation is performed. The difference between the metrics of both cases and the standard deviation of that difference are plotted:

$$
\begin{aligned}
& x_{\text {corr }_{\text {gain }}}=x \text { corr }_{\text {interpolated }}-x \text { corr }_{\text {original }} \\
& S S I M_{\text {gain }}=S S I M_{\text {interpolated }}-S S I M_{\text {original }}
\end{aligned}
$$

It can be appreciated that there is always an improvement when the data coming from the gyroscopes is interpolated before restoring the image. In some cases, this improvement can even restore some completely lost images.
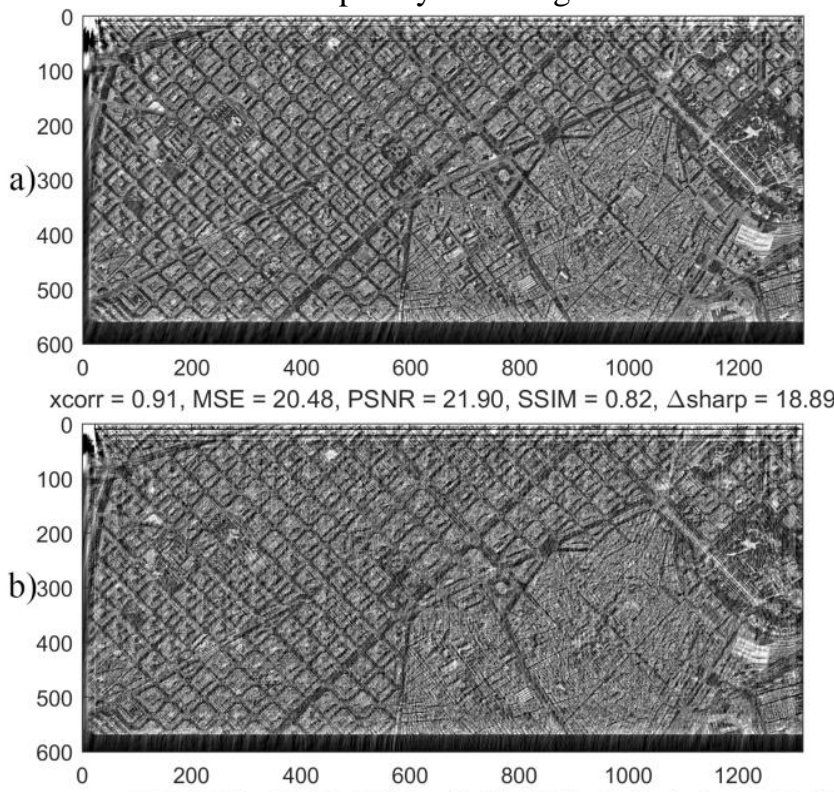

c)

xcorr $=0.63$, MSE $=39.20$, PSNR $=16.27$, SSIM $=0.43, \Delta$ sharp $=20.47$

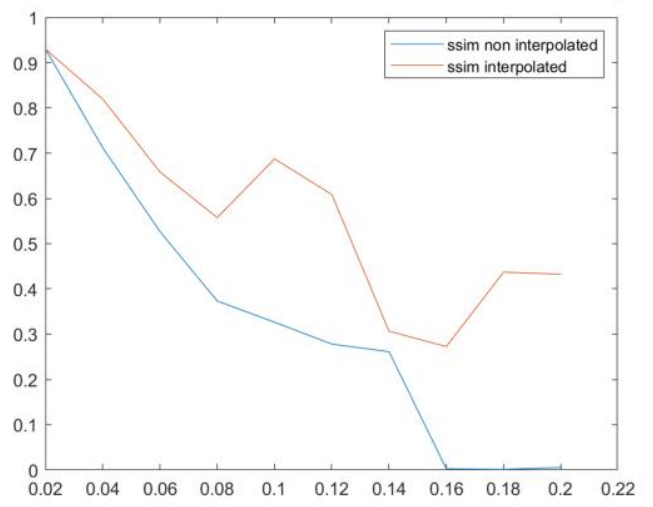

Figure 9. Temporal interpolation case D; (a) Restored image using interpolation from half temporal samples, (b) Restored image using interpolation from on tenth of the temporal samples, and (c) original cross-correlation and interpolated cross-correlation.

\section{CONCLUSIONS}

ADCSs are able to change the attitude of the satellite, but they have some drawbacks that are usually neglected, or at least not reported in the data sheets. The torque jitter created by the ADCS actuators induce non-desired rotation movements to the platform. This jitter combined with very high-resolution cameras results in blurred and useless images. However, the ADCS determination sensors are very useful because they can gather the data needed to infer the spatially variant PSF used by the image restoration methodology.

The temporal resolution of the data gathered by the sensors has also proved to be a key element in order to perform a good restoration. The presented deblurring technique performs better with more temporal samples, i.e. more image frames to compute the final image. In the case 
of coarse temporal samples, the image restoration can be improved using the interpolation of the data coming from the sensors.

The technique presented has been developed having in mind the current capabilities of imagers on board CubeSats. However, this technique is general, and it can be applied to very high-resolution imagers, onboard larger platforms with better pointing accuracies.

a)

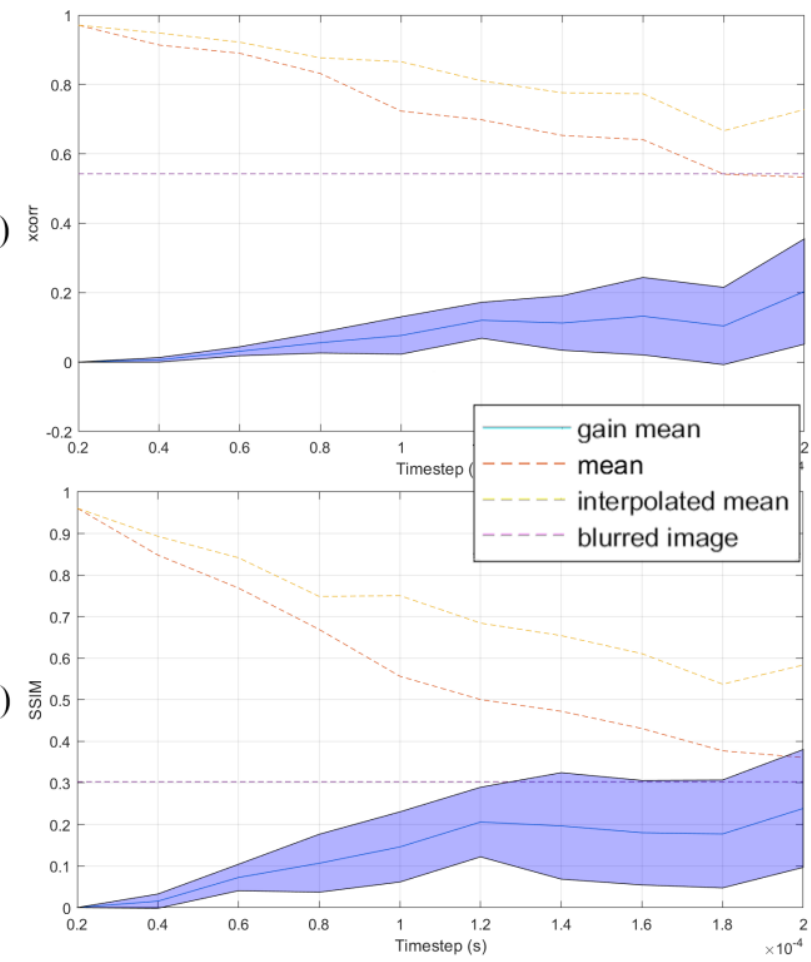

Figure 10. Interpolation performance improvement

\section{REFERENCES}

[1] Planet

Labs

Inc, https://www.planet.com/products/planet-imagery/

[Accessed 1301 2020].

[2] S. R. Starin and J. Eterno, "Attitude Determination and Control Systems," in Space mission engineering: the new SMAD, Microcosm Press, 2011, p. Ch 19.1.

[3] R. Stengel, "Spacecraft sensors and actuators," 2016. [Online].

Available: http://www.stengel.mycpanel.princeton.edu/MAE342Lectur e13.pdf. [Accessed 1410 2019].

[4] J. A. Ruiz-de-Azua et al., "3Cat-4 Mission: A 1-Unit CubeSat for Earth Observation with a L-band Radiometer and a GNSS-Reflectometer Using Software Defined Radio," IGARSS 2019 - 2019 IEEE International Geoscience and Remote Sensing Symposium, Yokohama, Japan, 2019, pp. 8867-8870.

[5] T. Inamori, J. Wang, P. Saisutjairt, S. Nakasuka, "Jitter reduction of a reaction wheel by management of angular momentum using magnetic torquers in nano- and microsatellites", Advances in Space Research, vol. 52, no. 1, pp. 222-231, 2013.
[6] C. Oh, W. Lee and H. Bang, "Passive Jitter Isolation for Reaction Wheel of Satellites," 2006 SICE-ICASE International Joint Conference, Busan, 2006, pp. 3891-3895. [7] ICGC "Institut Cartogràfic i Geològic de Catalunya," Generalitat de Catalunya. Url: http://www.icgc.cat/en/Downloads/Aerial-and-satelliteimages/Conventional-orthophoto. [Accessed 0210 2019]. [8] N. Joshi, S. B. Kang, C. L. Zitnik and R. Szeliski, "Image deblurring using inertial measurement sensors," ACM Transactions on Graphics (TOG), vol. 29, no. 4, pp. 30:130:9, 2010.

[9] C. Paige and M. Saunders, "LSQR: An Algorithm for Sparse Linear Equations and Sparse Least Squares," ACM Transactions on Mathematical Software, vol. 8, no. 1, pp. 4371, 1982.

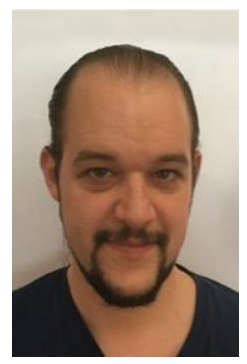

David Llaveria was born in Barcelona, Spain in 1984. He received the degree in Telecommunications Engineering and the master's degree in Telecommunications Engineering with Antennas, Microwaves and Photonics for Communications and Earth Observation specialization from the Universitat Politècnica de Catalunya (UPC), Barcelona, Spain, in 2017 and 2019 , respectively.

In 2016 he joined the UPC NanoSat Lab (http://www.tsc.upc.edu/nanosatlab) to work on the ONION project (http://www.onion-h2020.eu/). Since 2019 he is with the NanoSat Lab ${ }^{3}$ Cat -4 team, developing the attitude control software of the satellite and leading the ADCS test campaign. He is currently pursuing his $\mathrm{Ph} . \mathrm{D}$. in Telecommunications Engineering at the UPC at the NanoSat Lab, Barcelona, Spain. His research interests are focused in Earth Observation image processing, specially in hyperspectral images.

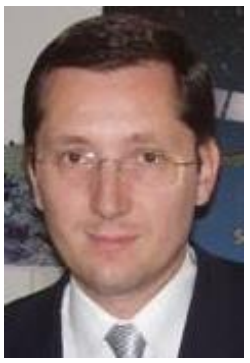

Prof. Adriano Camps (S'91-A'97-M'00-SM'03F'11) was born in Barcelona, Spain, in 1969. He received the degree in Telecommunications Engineering and a Ph.D. degree in Telecommunications Engineering from the Universitat Politècnica de Catalunya (UPC), Barcelona, Spain, in 1992 and 1996, respectively. In 1991 to 1992, he was at the ENS des Télécommunications de Bretagne, France, with an Erasmus Fellowship. Since 1993, he has been with the Electromagnetics and Photonics Engineering Group, Department of Signal Theory and Communications, UPC, where he was first Assistant Professor, Associate Professor in 1997, and Full Professor since 2007. In 1999, he was on sabbatical leave at the Microwave Remote Sensing Laboratory, of the University of Massachusetts, Amherst. Since 1993, he has been deeply involved in the European Space Agency SMOS Earth Explorer Mission, from the instrument and algorithmic points of view, performing field experiments, and since 2001 studying the use of GNSS-R techniques to perform the sea state correction needed to retrieve salinity from L-band radiometric observations. His research interests are focused in microwave remote sensing, with special emphasis in microwave radiometry by aperture synthesis techniques and remote sensing using signals of opportunity (GNSS-R). He has published over 203 papers in peerreviewed journals, 6 book chapters, 1 book, and more than 425 international conference presentations, holds 12 patents, and has advised $23 \mathrm{Ph}$. D. Thesis students (+ 8 on-going), and more than 120 final projects and M.Eng. Theses. According to Publish or Perish (Google Scholar) his publications have received more than 6895/10250 citations, and his h-index is $38 / 49$ according to Scopus/Google Scholar.

Prof. Adriano Camps is currently the Scientific Coordinator of the CommSensLab "María de Maeztu" Excellence Research Unit, he co-led the Remote Sensing Lab (www.tsc.upc.edu/rs, www.tsc.upc.edu/prsblog) and co-leads the UPC NanoSat Lab (http://www.tsc.upc.edu/nanosatlab). He is the PI of the first four UPC nano-satellites: 1) 3Cat-1, a 1U CubeSat with 7 small technology demonstrators and scientific payloads, 2) 3Cat-2, a $6 \mathrm{U}$ 
CubeSat with the first dual-frequency dual-polarization GNSS-R payload, launched on August 15th 2016 using a Chinese LM-D2 rocket, 3) 3Cat-4, a

$1 \mathrm{U}$ Cubesat with a software defined radio to implement a microwave radiometer, a GNSS-Reflectomer, and an AIS receiver, and 4) FSSCAT, a tandem mission formed by two $6 \mathrm{U}$ CubeSats, overall winner of the ESA 2017 Sentinel Small Satellite Challenge and Copernicus Masters competition in 2017 .

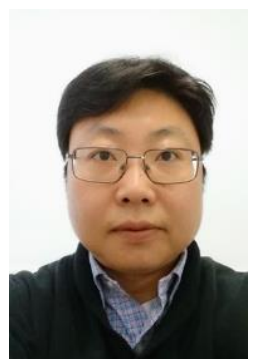

Hyuk Park (S'05-AM'09-M'12-SM'15) was born in South Korea. He received the B.S. degree in mechanical engineering from the Korea Advanced Institute of Science and Technology (KAIST), Daejeon, Korea, in 2001 and the M.S. and Ph.D. degrees in information and mechatronics from Gwangju Institute of Science and Technology (GIST), Gwangju, Korea, in 2003 and 2009, respectively. In 2009, he joined the Remote Sensing Group, Polytechnic University of Catalonia (UPC),

Barcelona, Spain, as a Postdoctoral Researcher. In 2011, he was a grant holder of National Research Foundation funded by the Korean Government. Since 2012, he has been working as a Research Associate with a Juan de la Cierva grant funded by the Spanish Ministry of Economy and Competitiveness. He is currently working with School of Telecommunications and Aerospace Engineering at Castelldefels (EETACUPC) as a Ramon y Cajal Fellow / Tenure-track Assistant Professor. He is also working with the Passive Remote Sensing Group, UPC, for satellite remote sensing for microwave radiometry and Global Navigation Satellite Systems reflectometry. His main research interest is in the area of remote sensing, particularly passive microwave remote sensing, including system design, modeling and simulation, image processing, and small satellite applications. 